\title{
The Expression of HMGB1 in Bone Marrow MSCs Is Upregulated by Hypoxia with Regulatory Effects on the Apoptosis and Adhesion
}

\author{
Mei-Yun Tan, ${ }^{1}$ Cai-Dong Zhang, ${ }^{1}$ Bo Xia, ${ }^{1}$ Jiang Guo, ${ }^{1}$ Zhong-Wei Fan, ${ }^{1}$ Tian-Hao Wu, \\ Sen Wang, ${ }^{1}$ Shao-Feng Liu, ${ }^{1}$ Li Deng, ${ }^{2}$ Xing Guo, ${ }^{3}$ and Yong-Can Huang ${ }^{4,5,6}$ \\ ${ }^{1}$ Department of Bone and Joint Surgery, The Affiliated Hospital of Southwest Medical University, Luzhou 646000, China \\ ${ }^{2}$ Laboratory of Stem Cell and Tissue Engineering, State Key Laboratory of Biotherapy, West China Hospital, \\ Sichuan University, Chengdu 610041, China \\ ${ }^{3}$ Department of Burns and Plastic Surgery, The Affiliated Hospital of Southwest Medical University, Luzhou 646000, China \\ ${ }^{4}$ Shenzhen Engineering Laboratory of Orthopaedic Regenerative Technologies, Orthopaedic Research Center, \\ Peking University Shenzhen Hospital, Shenzhen, China \\ ${ }^{5}$ Shenzhen Key Laboratory of Spine Surgery, Department of Spine Surgery, Peking University Shenzhen Hospital, Shenzhen, China \\ ${ }^{6}$ Department of Orthopaedics and Traumatology, Li Ka Shing Faculty of Medicine, The University of Hong Kong, \\ Pok Fu Lam 999077, Hong Kong
}

Correspondence should be addressed to Xing Guo; guo_xinglzmc@sina.com and Yong-Can Huang; y.c.huang@connect.hku.hk

Received 14 June 2016; Revised 24 September 2016; Accepted 13 October 2016

Academic Editor: Elena Jones

Copyright (C) 2016 Mei-Yun Tan et al. This is an open access article distributed under the Creative Commons Attribution License, which permits unrestricted use, distribution, and reproduction in any medium, provided the original work is properly cited.

Background and Aims. Hypoxia regulates the survival of mesenchymal stem cells (MSCs) but the mechanism is unclear. In hypoxia, the level of high mobility group box 1 (HMGB1) was increased in many cells which may be involved in the regulation of cell biology. The aim is to determine whether hypoxia affects the expression of HMGB1 in bone marrow MSCs (BM-MSCs) and to investigate the role of HMGB1 in the apoptosis and adhesion. Methods. BM-MSCs were exposed to hypoxia $\left(1 \% \mathrm{O}_{2}\right)$ and normoxia $\left(20 \% \mathrm{O}_{2}\right)$ and the expression of HMGB1 was measured by RT-PCR and western blotting. The apoptosis and adhesion of BM-MSCs were evaluated after interfered by different concentrations of HMGB1. Results. Expression of HMGB1 in BM-MSCs showed a significant upregulation in hypoxia when compared to those in normoxia. The adhesion of BM-MSCs was increased by HMGB1 in a concentration-dependent manner; the apoptosis effect of HMGB1 depended on its concentrations: HMGB1 at low concentration $(50 \mathrm{ng} / \mathrm{mL})$ promoted the apoptosis of BM-MSCs while HMGB1 at high concentration $(\geq 100 \mathrm{ng} / \mathrm{mL}) \mathrm{reduced}$ this apoptosis. Conclusions. Hypoxia enhanced the expression of HMGB1 in BM-MSCs with influences on apoptosis and adhesion and this could have a significant effect on the regenerative potential of MSC-based strategies.

\section{Introduction}

Mesenchymal stem cells (MSCs) are cells with the capacity of self-renewal and multiple differentiation abilities [1]. With the use of particular inducers, they can differentiate into osteocytes [2], chondrocytes [3], and other cells [4]. They are currently the most promising seed cells for tissue regeneration, having been successfully tested in treatment of many diseases such as degenerative diseases [5] and myocardial infarction [6] in humans. Nevertheless, the posttransplanted cells have to experience the hash microenvironment (hypoxia, low nutrition, inflammation, etc.) and most of them would die in the first 24 hours [7]. Quick loss of the implanted cells remains a big challenge for MSC-based therapies.

It was shown that hypoxic preconditioning could enhance the survival of MSCs after transplantation [8-10], but the underlying mechanism is still unknown. High mobility group box 1 (HMGB1), a nonhistone chromosomal binding protein, is released into extracellular space by damaged cells, necrotic cells, and activated inflammatory cells [11]. The biological 
TABLE 1: Specific primers used for real-time polymerase chain reaction.

\begin{tabular}{llr}
\hline Primer name & \multicolumn{1}{c}{ Primer sequences } & Fragment length \\
\hline \multirow{2}{*}{ HMGB1 } & Forward: $5^{\prime}$-GGCGGCTGTTTTGTTGACAT-3' & 135 bp \\
& Reverse: $5^{\prime}$-ACCCAAAATGGGCAAAAGCA-3' & \\
GAPDH & Forward: $5^{\prime}$-ACCACAGTCCATGCCATCAC-3' & 452 bp \\
& Reverse: $5^{\prime}$-TCCACCACCCTGTTGCTGTA-3' & \\
\hline
\end{tabular}

function of these cells will be changed by HMGB1 with its subsequent binding to RAGE, toll-like receptors, and other membrane receptors $[12,13]$. Studies have shown that hypoxia can induce the expression of HMGB1 in many kinds of cells such as chondrogenic ATDC5 cells, synovial fibroblast cells, monocyte/macrophage-lineage cell lines (HL-60 and U937) [14], and HTLA-230 cells [15]; interestingly, there was almost no expression in these cells under normoxic condition. Nevertheless, the effect of hypoxia on the expression of HMGB1 in BM-MSCs was still unclear. Thus, the first aim of this study was to investigate the expression level of HMGB1 in hypoxiccultured BM-MSCs. Additionally, several researchers and our group have found that HMGB1 could significantly inhibit the proliferation and enhance the migration of BM-MSCs $[16,17]$. But no finding regarding the regulation effects of HMGB1 in the apoptosis and adhesion of BM-MSCs was reported. Hence, the second aim of this work was to unravel the effect of HMGB1 on the apoptosis and the adhesive ability of BMMSCs in vitro.

Therefore, in this study, BM-MSCs were isolated from rats and exposed to hypoxia; the expression of HMGB1 was then measured in vitro by RT-PCR and western blotting analysis. On the other hand, the effects of HMGB1 on the apoptosis and the adhesion of BM-MSCs were investigated by flow cytometry and Fibronectin-coating adhesives assessment, respectively.

\section{Materials and Methods}

2.1. Isolation and Identification of BM-MSCs. Fifteen Sprague-Dawley rats weighing 150-200 g were supplied by the Experimental Animal Center of Southwest Medical University and the experimental procedures were approved by local Laboratory Animal Ethics Committee. BM-MSCs were isolated according to our previous description [18]. Briefly, rat bone marrow was collected by washing the femurs and tibias with Phosphate Buffered Saline (PBS) and seeded into flasks with Dulbecco's Modified Eagle's medium (DMEM) supplemented with $10 \%$ fetal bovine serum (FBS) and $1 \%$ antibiotic/antimycotic solution (Gibco). The bone marrow cells were cultured at $37^{\circ} \mathrm{C}$ in a humidified incubator with $5 \% \mathrm{CO}_{2}$. To remove the nonadherent cells, the medium was replaced after $24 \mathrm{~h}$ and continued refreshing every 2-3 days. When the cells became $80 \%-90 \%$ confluent, they were subcultured at 1:2 dilution. In the flow cytometric analysis, the cells were identified with directly conjugated antibodies against anti-CD29, anti-CD31, anti-CD90, and anti-CD45 (BD Pharmingen, USA). BM-MSCs at passages 3-5 were used for all the experiments.
2.2. Experimental Hypoxic Condition. Hypoxic culture was performed when BM-MSCs of 3rd passages became $80 \%-$ $90 \%$ confluence. The cells were incubated in hypoxia incubator chambers (Thermo, USA) at $37^{\circ} \mathrm{C}$ in the presence of $1 \% \mathrm{O}_{2}$ and $5 \% \mathrm{CO}_{2}$. Normoxic control was incubated in a humidified chamber containing $20 \% \mathrm{O}_{2}$ and $5 \% \mathrm{CO}_{2}$. To compare the differences of the expression of HMGB1 between hypoxic and normoxic cultures, cells cultured for 24 hours were harvested for RT-PCR and western blot analysis.

2.3. RT-PCR. Total RNA was extracted and one microgram of RNA was reverse-transcribed into cDNA using isoPLUS reagent (TAKARA, Japan). The expression of HMGB1 mRNA was analyzed by RT-PCR. In brief, the specific primer sequences of HMGB1 were designed and synthesized (Table 1) and the amplification conditions were listed as follows: denaturing at $95^{\circ} \mathrm{C}$ for $30 \mathrm{~s}$ followed by 40 cycles at $95^{\circ} \mathrm{C}$ for $15 \mathrm{~s}$ and $60^{\circ} \mathrm{C}$ for $30 \mathrm{~s}$. Then the PCR reactions were separated by $1 \%$ agarose gel electrophoresis. All procedures were repeated three times in triplicate.

2.4. Western Blotting. Western blotting was performed to assess HMGB1 accumulation in BM-MSCs after being exposed to hypoxia. Whole-cell protein lysates were collected on ice. The protein $(20 \mu \mathrm{g})$ of each sample was separated on a $10 \%$ SDS-PAGE gel (Invitrogen, USA) and electrotransferred to nitrocellulose membranes (Amersham Pharmacia Biotech, USA). After the membranes were blocked in TBST containing $5 \%$ nonfat milk, the blots were incubated with HMGB1 primary antibody (Cell Signaling Technology, USA) (1:1000) overnight at $4^{\circ} \mathrm{C}$. The immunoreactive bands were visualized using chemiluminescence regent kit (Sigma, USA) after the membranes incubated with HRP-conjugated secondary antibody. Signals from the bands were analyzed by an imaging densitometer (HP, USA). All procedures were repeated three times in triplicate.

2.5. Apoptosis Assessment. The method used for apoptosis assessment was similar to our previous description [19]. Briefly, $2 \times 10^{5}$ cells were planted in 12 -well plates and incubated for $24 \mathrm{~h}$ at $37^{\circ} \mathrm{C}$ incubator. Then, the initial solutions were replaced with serum-free DMEM for serum deprivation (SD) culture. HMGB1 with different concentrations $(0,10$, 50,100 , and $200 \mathrm{ng} / \mathrm{mL}$ ) were added at the same time after serum deprivation for cell apoptosis assays ( $n=5$, triplicate). After 24 hours of HMGB1 interfering, the cell apoptosis was assessed using the Annexin V-FITC apoptosis detection kit (BD Pharmingen, USA). Briefly, the cells were collected, washed, and resuspended in binding buffer; to stain the 


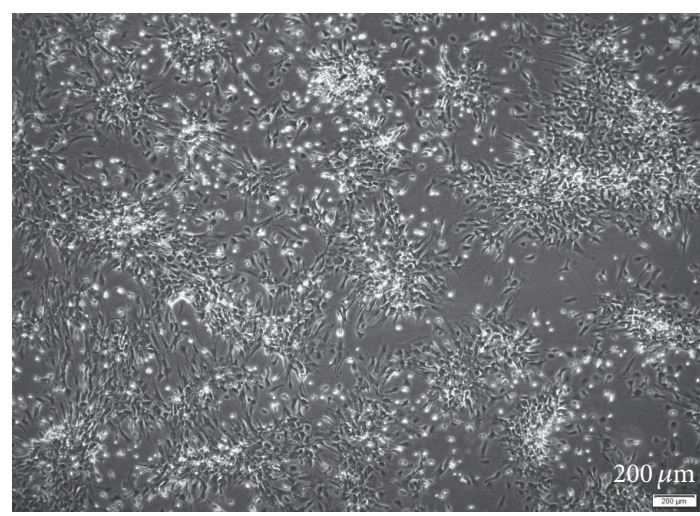

(a)

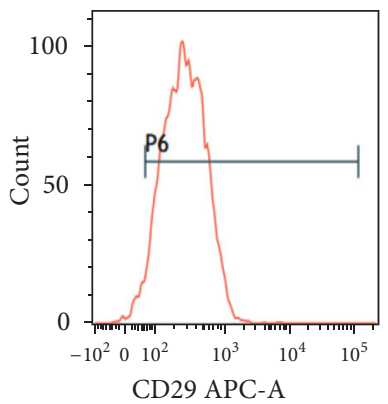

CD29 APC-A

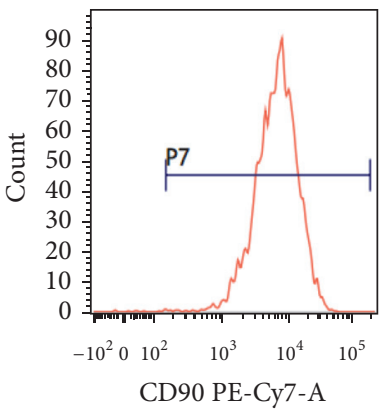

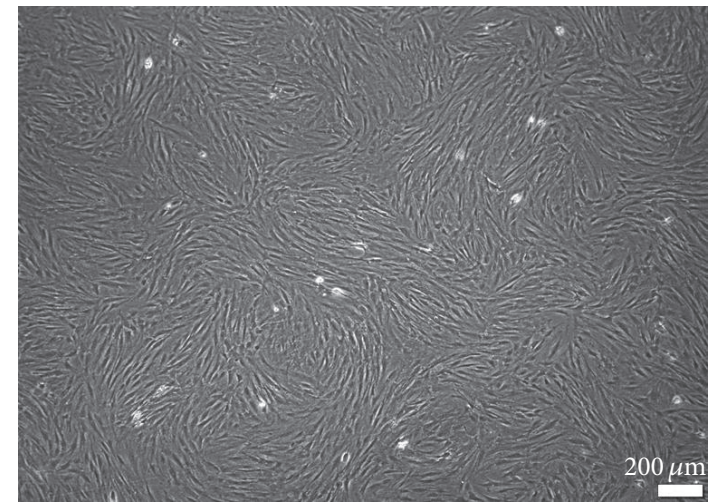

(b)
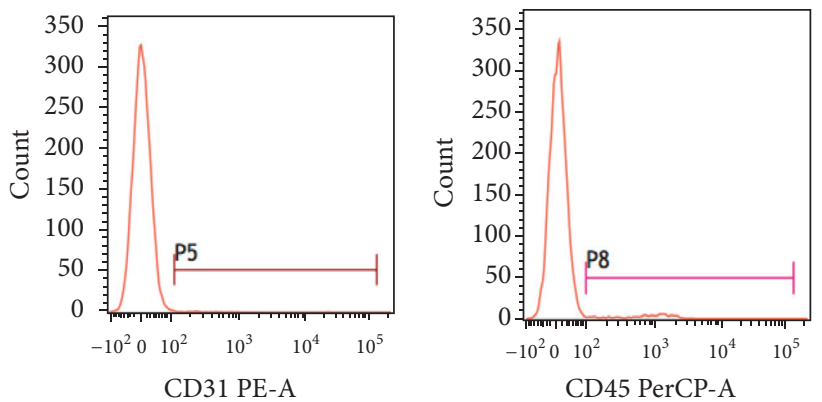

(c)

Figure 1: Characterization of BM-MSCs. (a) The $\mathrm{P}_{0}$ cells grew in colonies after being cultured for 5-6 days. (b) Representative images of $\mathrm{P}_{3}$ BM-MSCs (original magnification, $\times 40$ ); the cells showed typically spindle-shape morphology. (c) Flow cytometric analysis of cell surface marker expression of the isolated cells. The cells were positive for MSCs specific markers CD29 and CD90 but negative for hematopoietic markers CD31 and CD45.

apoptotic cells, Annexin V-FITC and propidium iodide were added followed by $15 \mathrm{~min}$ reaction at room temperature. Then, the stained cells were subjected to flow cytometry using a FACScan flow cytometer (BD Biosciences, USA).

2.6. Cell Adhesion Assessment. The adhesion of BM-MSCs was evaluated according to previous report [8]. Briefly, Fibronectin (FN) (Prospec, Israel) with a concentration of $1 \mu \mathrm{g} / \mathrm{cm}^{2}$ was used as a thin coating on 12 -well plate surface overnight, and the supernatant solution was removed. BMMSCs were seeded at $5 \times 10^{4}$ cells/well in the 12 -well plate, followed by $24 \mathrm{~h}$ cultivation with HMGB1 at different concentrations $(0,10,50,100$, and $200 \mathrm{ng} / \mathrm{mL})(n=5)$. Cells seeded in the complete medium without FN were used as control. After 24 hours, the nonadherent cells were removed by washing with PBS for 30 seconds in the orbital shaker. Then the cells cohered to FN were counted in five random microscopic fields (100-fold magnification) in each well and the mean value of each well was measured to reflect the adhesion level. Experiments were repeated 3 times.

2.7. Statistical Analysis. Data were analyzed using SPSS 19.0 (SPSS 19.0 Inc., Chicago, IL, USA). One-way analysis of variance (ANOVA) followed by LSD-test was used to determine the significance of difference of all statistical data. Significance was accepted as $P<0.05$.

\section{Results}

3.1. Characterization of BM-MSCs. Cells were successfully isolated from the bone marrow. After 5-6 days of culture, the adherent cells grew in colonies (Figure $1(\mathrm{a})$ ); cells at $\mathrm{P}_{3}$ (the third passage) showed a typical spindle-shaped appearance and arranged radially (Figure 1(b)). Flow cytometric analysis results indicated that the cells were positive for CD29 and CD90 (>95\%) and negative for CD31 and CD45 (<5\%) (Figure 1(c)).

3.2. Effect of Hypoxia on the Expression of HMGB1 in BMMSCs. RT-PCR data showed that 24-hour hypoxia exposure led to an obvious increase of the expression of HMGB1 mRNA in BM-MSCs when compared to cells in normoxia $(P<0.05)$ (Figures 2(a) and 2(b)). Consistent with the increase of mRNA levels, it was confirmed by western blotting experiment showing the higher levels of HMGB1 protein in BM-MSCs under hypoxia $(P<0.05)$ (Figures $2(\mathrm{c})$ and $2(\mathrm{~d}))$. Hence, the expression level of HMGB1 in BM-MSCs was notably increased by hypoxia at gene and protein levels.

3.3. Effect of HMGB1 on the Apoptosis of BM-MSCs. MSCs cultured in the absence or presence of different concentrations of HMGB1 $(10,50,100$, and $200 \mathrm{ng} / \mathrm{mL})$ were exposed to serum deprivation (SD) for 24 hours. The apoptotic 


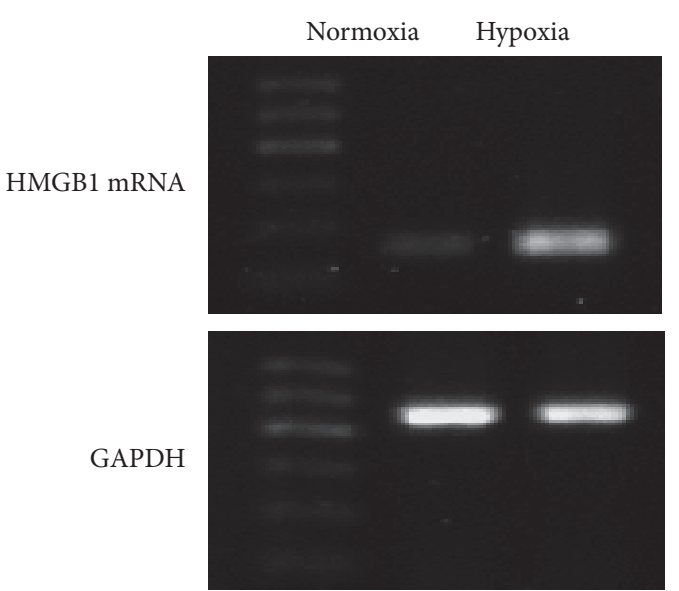

(a)

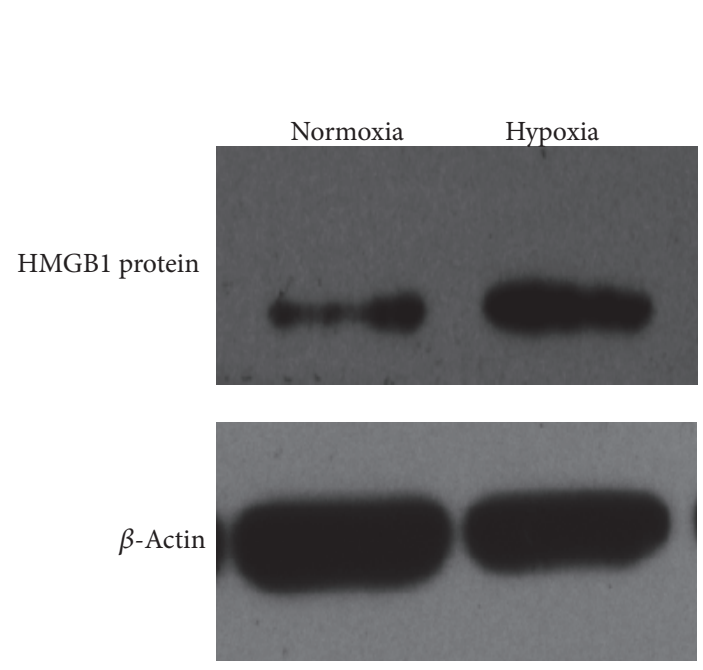

(c)

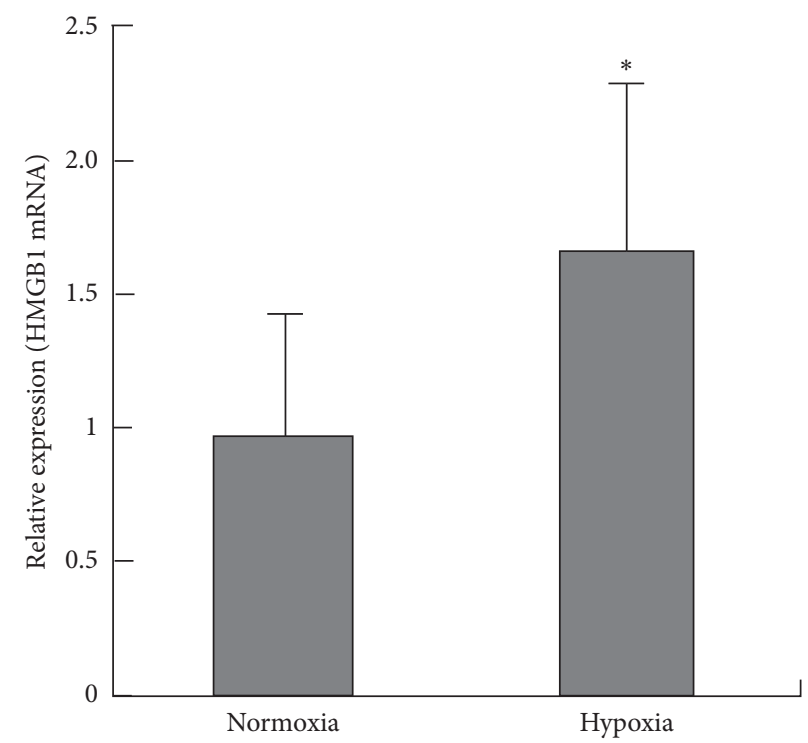

(b)

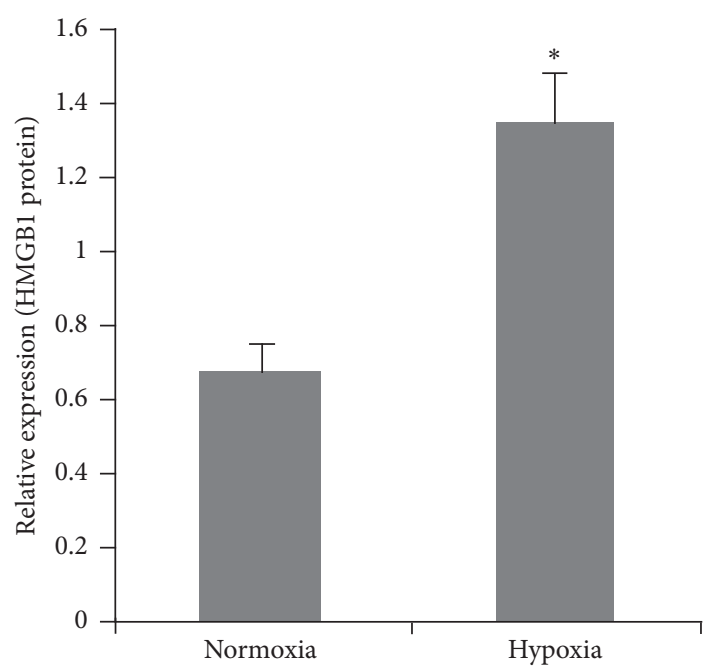

(d)

FIGURE 2: Effect of hypoxia on the expression of HMGB1 in BM-MSCs. Cells were exposed to hypoxic $\left(1 \% \mathrm{O}_{2}\right)$ or normoxic conditions for $24 \mathrm{~h}$. HMGB1 at mRNA and protein levels were detected by RT-PCR ((a)-(b)) and western blotting ((c)-(d)), respectively. GAPDH and $\beta$-actin were used as the control. The data was shown as mean $\pm \operatorname{SEM}(n=3) .{ }^{*} P<0.05$ versus normoxia.

rate was measured by FACS analysis using Annexin V/PI staining. Increased apoptosis rate of BM-MSCs was observed after HMGB1 treatment (Figure 3(a)). Compared with the single SD culture group (0 ng/mL HMGB1, $9.03 \pm 0.93)$, the apoptotic rate of BM-MSCs was much higher when the HMGB1 concentrations were $50 \mathrm{ng} / \mathrm{mL}(18.23 \pm 1.74)$ and $100 \mathrm{ng} / \mathrm{mL}(17.13 \pm 2.89)(P<0.05)$. However, the apoptotic rate was not changed at the HMGB1 treatment group with concentration of $10 \mathrm{ng} / \mathrm{mL}(10.02 \pm 2.08)$ and $200 \mathrm{ng} / \mathrm{mL}$ $(13.97 \pm 2.62)$, when compared with the single SD group (Figure 3(b)).

3.4. HMGB1 Increased the Adhesion of BM-MSCs. To investigate the effects of HMGB1 on the adhesion of BMMSCs, cells were seeded in 12-well plate which was coated with FN overnight, followed by 24 hours of $0-200 \mathrm{ng} / \mathrm{mL}$ HMGB1 treatments. With the increasing concentration of HMGB1, the number of adherent BM-MSCs was increased apparently (Figure 4(a)). Quantitative analysis showed that HMGB1 treatment groups (FN + $50 \mathrm{ng} / \mathrm{mL}: 245 \pm 16.3$; FN $+100 \mathrm{ng} / \mathrm{mL}: 267.6 \pm 1.0 ; \mathrm{FN}+200 \mathrm{ng} / \mathrm{mL}: 304.0 \pm 19.1)$ obviously increased the number of adhesive BM-MSCs when compared to single FN culture group (FN + $0 \mathrm{ng} / \mathrm{mL}, 194.4 \pm$ 18.3) after $24 \mathrm{~h}$ treatment $(P<0.05)$ (Figure $4(\mathrm{~b}))$.

\section{Discussion}

This study provided evidence that hypoxia was able to induce significant upregulation of HMGB1 in BM-MSCs; 


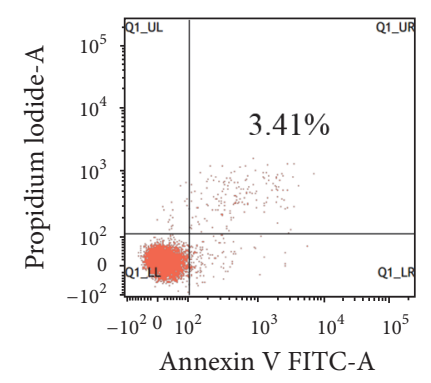

Control

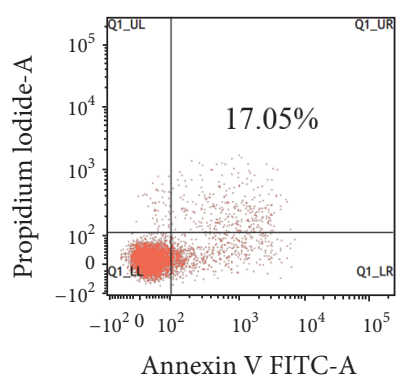

$\mathrm{SD}+50$ ng/mL HMGB1

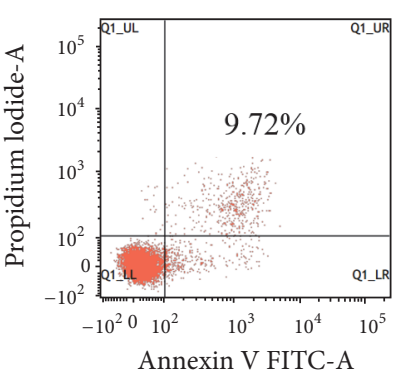

$\mathrm{SD}+0 \mathrm{ng} / \mathrm{mL}$ HMGB1

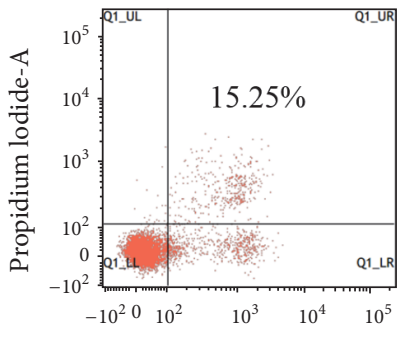

Annexin V FITC-A

$\mathrm{SD}+100 \mathrm{ng} / \mathrm{mL}$ HMGB1

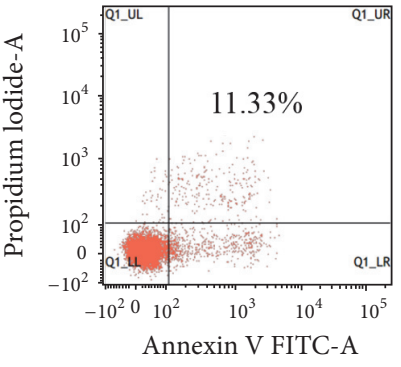

$\mathrm{SD}+10 \mathrm{ng} / \mathrm{mL}$ HMGB1

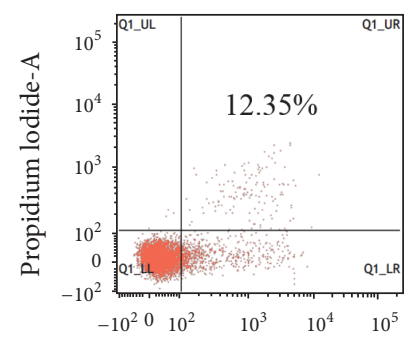

Annexin V FITC-A

SD + 200 ng/mL HMGB1

(a)

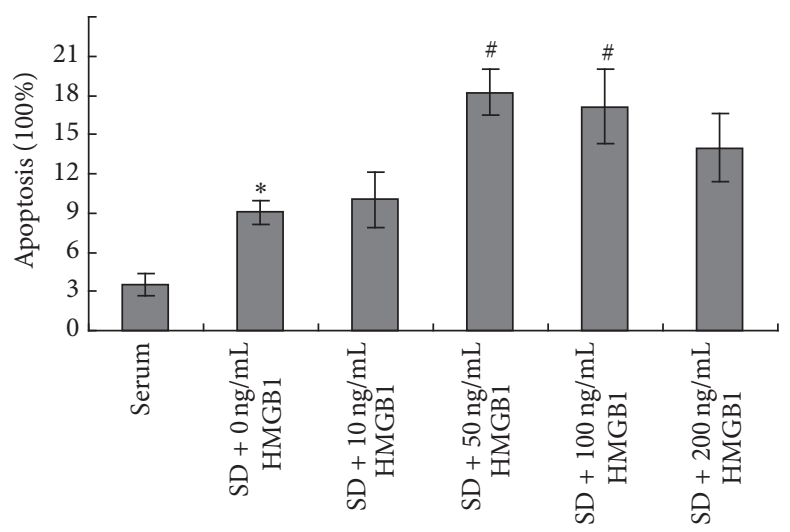

(b)

FIGURE 3: Effect of HMGB1 on the apoptosis of BM-MSCs. (a) Cells with different concentration of HMGB1 (0, 10, 50, 100, and 200 ng/mL) were exposed to serum deprivation (SD) for 24 hours. Apoptotic rate was calculated by FACS analysis of Annexin V/PI staining and the representative images of apoptotic cell populations were illustrated. (b) Data was shown as mean $\pm \operatorname{SEM}(n=5) .{ }^{*} P<0.05$ versus serum(s) group. ${ }^{\#} P<0.05$ versus the group of cells exposed to SD with $0 \mathrm{ng} / \mathrm{mL}$ HMGB1.

then it further demonstrated that HMGB1 could promote the adhesion and regulate the apoptosis of BM-MSCs in vitro.

Oxygen concentration plays a crucial role in regulating the biology of BM-MSCs [20]. Previous studies show that hypoxia could influence the proliferation, migration, adhesion, differentiation, apoptosis, and angiogenesis ability of progenitor cells $[8,21,22]$. It is well known that HIF$1 \alpha$ is a key regulatory transcription factor during hypoxia [23], but researchers have never paid an equal attention to the effect of HMGB1 on MSCs under hypoxia. Recently, it was found that hypoxia or ischemia increased the levels of HMGB1 in many kinds of cells [14, 15]. Additionally, HMGB1 regulated the proliferation and migration of BMMSCs [16, 17]. Considering these findings, we hypothesized that HMGB1 may also play an important role in regulating the behaviors of BM-MSCs in hypoxia. Hence, we compared the expression levels of HMGB1 in BM-MSCs under hypoxia and normoxia.

Many researches showed that HMGB1 conjugated with TLR or RAGE receptors of BM-MSCs which subsequently triggered the inflammatory reaction and vascular formation $[12,13,24]$. Nevertheless, little is known regarding its role in regulating biology of BM-MSCs. Cell adhesion is an important character for cell movement and tissue separation $[25,26]$. It was reported that HMGB1 could improve the adhesion of endothelial cells [27, 28], bEnd 3 cell line [29], and c-kit ${ }^{+}$cells [30]. In this study, we further explored the effect of HMGB1 on the adhesion of BM-MSCs and concluded that 


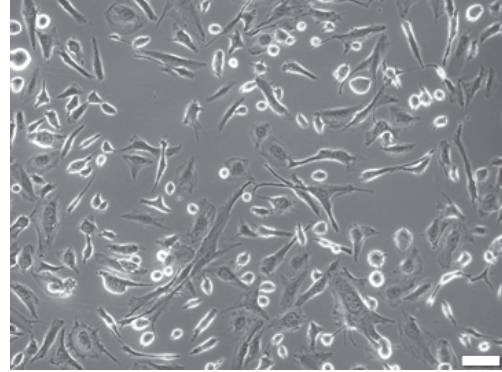

Non-FN

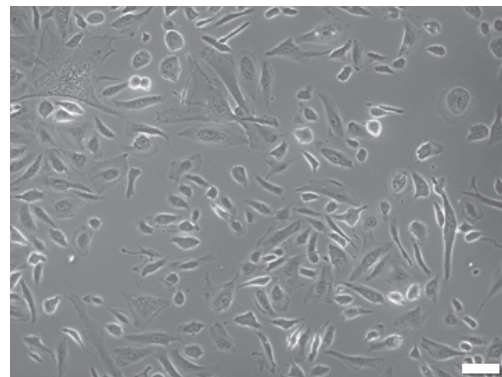

$\mathrm{FN}+50 \mathrm{ng} / \mathrm{mL}$ HMGB1

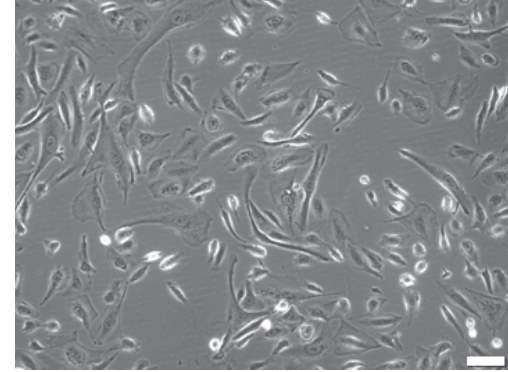

$\mathrm{FN}+0 \mathrm{ng} / \mathrm{mL}$ HMGB1

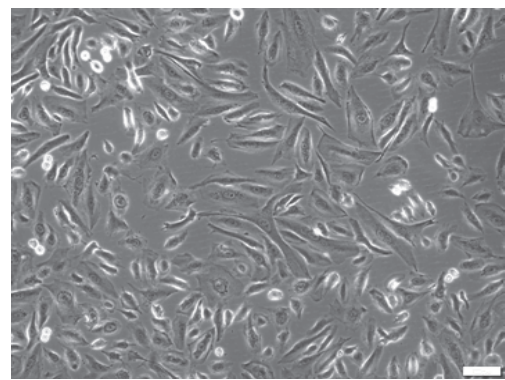

$\mathrm{FN}+100 \mathrm{ng} / \mathrm{mL}$ HMGB1

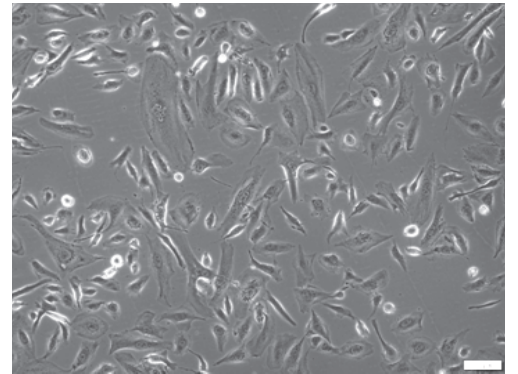

$\mathrm{FN}+10 \mathrm{ng} / \mathrm{mL}$ HMGB1

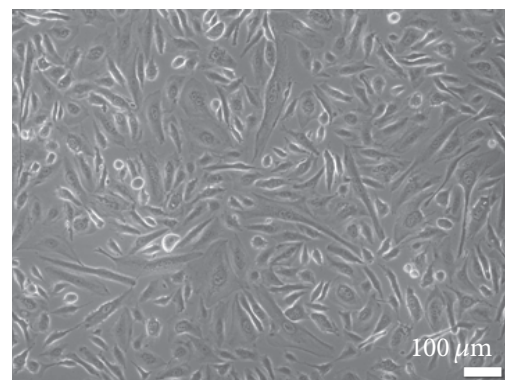

$\mathrm{FN}+200 \mathrm{ng} / \mathrm{mL}$ HMGB1

(a)

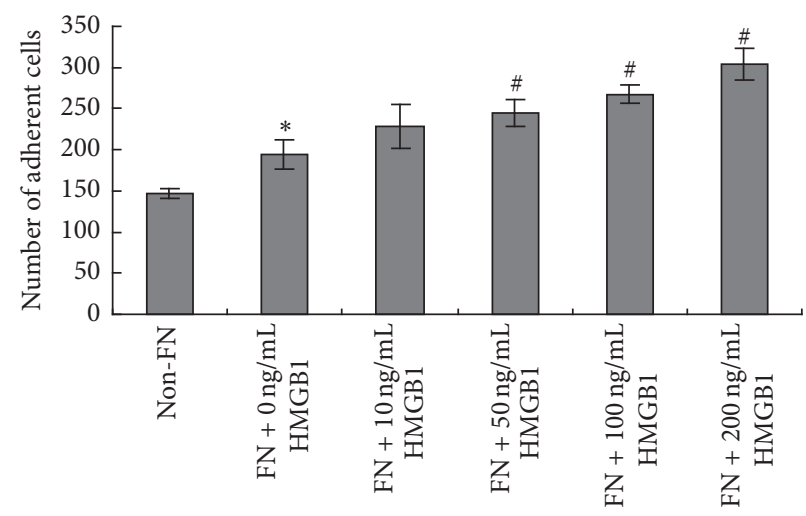

(b)

FIGURE 4: Effect of HMGB1 on the adhesion of BM-MSCs. (a) BM-MSCs were cultured with HMGB1 at the concentrations of 0, 10, 50, 100, and $200 \mathrm{ng} / \mathrm{mL}$ for $24 \mathrm{~h}$; the representative images of BM-MSCs under phase-contrast microscopy (original magnification, $\times 100$ ) were taken after the nonadherent cells were removed. (b) Quantification of adhesive cells. Data was shown as mean \pm SEM $(n=5) .{ }^{*} P<0.05$ versus non-FN group. ${ }^{*} P<0.05$ versus " $\mathrm{FN}+0 \mathrm{ng} / \mathrm{mL} \mathrm{HMGB1}$ " group.

HMGB1 was able to increase the adhesion of BM-MSCs in a concentration-dependent manner.

Furthermore, our study indicated that HMGB1 was able to accelerate the apoptosis of BM-MSCs, especially at the concentration of $50 \mathrm{ng} / \mathrm{mL}$, but this increase tendency of apoptosis did not exist anymore when its concentration exceeded $100 \mathrm{ng} / \mathrm{mL}$. It was pointed out that HMGB1 alone or together with hypoxia upregulated the expression of HIF$1 \alpha$ in BM-MSCs [16] and HIF- $1 \alpha$ was helpful to protect BMMSCs from serum deprivation induced apoptosis [31]. After HMGB1 (100 ng/mL) stimulation, the expression of HIF-1 $\alpha$ mRNA and protein were notably increased in RA fibroblasts [32]. The higher concentration of HMGB1 may lead to higher expression level of HIF-1 $\alpha$ against apoptosis in BM-MSCs, which may be a possible explanation for the phenomenon that $100 \mathrm{ng} / \mathrm{mL}$ did not induce higher apoptosis than the lower concentration of HMGB1 in our study. Hence, HMGB1 had the potential to accelerate the apoptosis of BM-MSCs, but it may also have a protective effect against cell apoptosis via the activation of HIF- $1 \alpha$.

Nevertheless, there are two major limitations that we should acknowledge in this work. First, the relationship between HIF- $1 \alpha$ and HMGB1 in BM-MSCs was not well understood; future investigation using transgenic mouse model will be extremely meaningful to unravel this important issue. Second, further experiments are needed to understand the detailed mechanism concerning the regulation of HMGB1 in the adhesion and in the apoptosis of BM-MSCs. It can be 
presumed that there should be a HIF-1 $\alpha$-HMGB1 pathway to enhance the survival of postimplanted BM-MSCs. Definitely, the therapeutic potential of HMGB1 cannot be ignored, especially in the field of MSC-based therapies.

\section{Competing Interests}

The authors declare that there is no conflict of interests.

\section{Authors' Contributions}

Mei-Yun Tan, Cai-Dong Zhang, Xing Guo, and YongCan Huang contributed to the experimental design, data acquisition, analysis, and interpretation. Mei-Yun Tan, CaiDong Zhang, Xing Guo, and Yong-Can Huang drafted the manuscript. Bo Xia, Jiang Guo, Zhong-Wei Fan, Tian-Hao $\mathrm{Wu}$, Sen Wang, Shao-Feng Liu, and Li Deng provided technical support and assisted with the data analysis and interpretation. All the authors approved the final manuscript to be submitted. Mei-Yun Tan and Cai-Dong Zhang contributed equally to this work.

\section{Acknowledgments}

This work was financially supported by the grants (nos. 31271049 and 31570970 ) from the National Science Foundation of China.

\section{References}

[1] C. Nesselmann, N. Ma, K. Bieback et al., "Mesenchymal stem cells and cardiac repair," Journal of Cellular and Molecular Medicine, vol. 12, no. 5B, pp. 1795-1810, 2008.

[2] T. J. Heino and T. A. Hentunen, "Differentiation of osteoblasts and osteocytes from mesenchymal stem cells," Current Stem Cell Research \& Therapy, vol. 3, no. 2, pp. 131-145, 2008.

[3] R. F. Pereira, K. W. Halford, M. D. O’Hara et al., "Cultured adherent cells from marrow can serve as long-lasting precursor cells for bone, cartilage, and lung in irradiated mice," Proceedings of the National Academy of Sciences of the United States of America, vol. 92, no. 11, pp. 4857-4861, 1995.

[4] C. Toma, M. F. Pittenger, K. S. Cahill, B. J. Byrne, and P. D. Kessler, "Human mesenchymal stem cells differentiate to a cardiomyocyte phenotype in the adult murine heart," Circulation, vol. 105, no. 1, pp. 93-98, 2002.

[5] P. A. Sotiropoulou, S. A. Perez, M. Salagianni, C. N. Baxevanis, and M. Papamichail, "Characterization of the optimal culture conditions for clinical scale production of human mesenchymal stem cells," Stem Cells, vol. 24, no. 2, pp. 462-471, 2006.

[6] A. R. Williams, K. E. Hatzistergos, B. Addicott et al., "Enhanced effect of combining human cardiac stem cells and bone marrow mesenchymal stem cells to reduce infarct size and to restore cardiac function after myocardial infarction," Circulation, vol. 127, no. 2, pp. 213-223, 2013.

[7] Y. L. Tang, Y. Tang, Y. C. Zhang, K. Qian, L. Shen, and M. I. Phillips, "Improved graft mesenchymal stem cell survival in ischemic heart with a hypoxia-regulated heme oxygenase-1 vector," Journal of the American College of Cardiology, vol. 46, no. 7, pp. 1339-1350, 2005.
[8] H. Liu, W. Xue, G. Ge et al., "Hypoxic preconditioning advances CXCR4 and CXCR7 expression by activating HIF-1 $\alpha$ in MSCs," Biochemical and Biophysical Research Communications, vol. 401, no. 4, pp. 509-515, 2010.

[9] N. Haque, M. T. Rahman, N. H. Abu Kasim, and A. M. Alabsi, "Hypoxic culture conditions as a solution for mesenchymal stem cell based regenerative therapy," The Scientific World Journal, vol. 2013, Article ID 632972, 12 pages, 2013.

[10] Y. C. Hung, O. Parolini, L. Deng et al., "Should hypoxia preconditioning become the standardized procedure for bone marrow MSCs preparation for clinical use?" Stem Cells, vol. 34, no. 7, pp. 1992-1993, 2016.

[11] P. Scaffidi, T. Misteli, and M. E. Bianchi, "Release of chromatin protein HMGB1 by necrotic cells triggers inflammation," Nature, vol. 418, no. 6894, pp. 191-195, 2002.

[12] G. Dvoriantchikova, E. Hernandez, J. Grant, A. R. C. Santos, H. Yang, and D. Ivanov, "The high-mobility group box-1 nuclear factor mediates retinal injury after ischemia reperfusion," Investigative Ophthalmology and Visual Science, vol. 52, no. 10, pp. 7187-7194, 2011.

[13] J. R. van Beijnum, W. A. Buurman, and A. W. Griffioen, "Convergence and amplification of toll-like receptor (TLR) and receptor for advanced glycation end products (RAGE) signaling pathways via high mobility group B1 (HMGB1)," Angiogenesis, vol. 11, no. 1, pp. 91-99, 2008.

[14] T. Hamada, M. Torikai, A. Kuwazuru et al., "Extracellular high mobility group box chromosomal protein 1 is a coupling factor for hypoxia and inflammation in arthritis," Arthritis and Rheumatism, vol. 58, no. 9, pp. 2675-2685, 2008.

[15] A. Pezzolo, D. Marimpietri, L. Raffaghello et al., "Failure of anti tumor-derived endothelial cell immunotherapy depends on augmentation of tumor hypoxia," Oncotarget, vol. 5, no. 21, pp. 10368-10381, 2014.

[16] H.-L. Xie, Y. Zhang, Y.-Z. Huang et al., "Regulation of high mobility group box 1 and hypoxia in the migration of mesenchymal stem cells," Cell Biology International, vol. 38, no. 7, pp. 892897, 2014.

[17] E. Meng, Z. Guo, H. Wang et al., "High mobility group box 1 protein inhibits the proliferation of human mesenchymal stem cells and promotes their migration and differentiation along osteoblastic pathway," Stem Cells and Development, vol. 17, no. 4, pp. 805-813, 2008.

[18] X. Guo, B. Xia, X.-B. Lu et al., "Grafting of mesenchymal stem cell-seeded small intestinal submucosa to repair the deep partial-thickness burns," Connective Tissue Research, vol. 57, no. 5, pp. 388-397, 2016.

[19] Y.-C. Huang, Z.-M. Yang, X.-H. Chen et al., "Isolation of mesenchymal stem cells from human placental decidua basalis and resistance to hypoxia and serum deprivation," Stem Cell Reviews and Reports, vol. 5, no. 3, pp. 247-255, 2009.

[20] A. Mohyeldin, T. Garzón-Muvdi, and A. Quiñones-Hinojosa, "Oxygen in stem cell biology: a critical component of the stem cell niche," Cell Stem Cell, vol. 7, no. 2, pp. 150-161, 2010.

[21] C. W. Pugh and P. J. Ratcliffe, "Regulation of angiogenesis by hypoxia: Role of the HIF system," Nature Medicine, vol. 9, no. 6, pp. 677-684, 2003.

[22] Y.-C. Huang, H.-M. Zhu, J.-Q. Cai et al., "Hypoxia inhibits the spontaneous calcification of bone marrow-derived mesenchymal stem cells," Journal of Cellular Biochemistry, vol. 113, no. 4, pp. 1407-1415, 2012. 
[23] A. J. Majmundar, W. J. Wong, and M. C. Simon, "Hypoxiainducible factors and the response to hypoxic stress," Molecular Cell, vol. 40, no. 2, pp. 294-309, 2010.

[24] Y. Ikegami, S. Miyoshi, N. Nishiyama et al., "Serumindependent cardiomyogenic transdifferentiation in human endometrium-derived mesenchymal cells," Artificial Organs, vol. 34, no. 4, pp. 280-288, 2010.

[25] X. Zhu, R. Bouffanais, and D. K. P. Yue, "Interplay between motility and cell-substratum adhesion in amoeboid cells," Biomicrofluidics, vol. 9, no. 5, Article ID 054112, 2015.

[26] R. Winklbauer, "Cell adhesion strength from cortical tensionan integration of concepts," Journal of Cell Science, vol. 128, no. 20, pp. 3687-3693, 2015.

[27] C. J. Treutiger, G. E. Mullins, A.-S. M. Johansson et al., "High mobility group 1 B-box mediates activation of human endothelium," Journal of Internal Medicine, vol. 254, no. 4, pp. 375-385, 2003.

[28] A. R. C. Santos, G. Dvoriantchikova, Y. Li et al., "Cellular mechanisms of high mobility group 1 (HMGB-1) protein action in the diabetic retinopathy," PLoS ONE, vol. 9, no. 1, Article ID e87574, 2014.

[29] J. Qiu, M. Nishimura, Y. Wang et al., "Early release of HMGB1 from neurons after the onset of brain ischemia," Journal of Cerebral Blood Flow and Metabolism, vol. 28, no. 5, pp. 927-938, 2008.

[30] D. Furlani, P. Donndorf, I. Westien et al., "HMGB-1 induces ckit+ cell microvascular rolling and adhesion via both toll-like receptor-2 and toll-like receptor-4 of endothelial cells," Journal of Cellular and Molecular Medicine, vol. 16, no. 5, pp. 1094-1105, 2012.

[31] A. A. Kiani, A. Kazemi, R. Halabian, M. Mohammadipour, A. Jahanian-Najafabadi, and M. H. Roudkenar, "HIF-lalpha confers resistance to induced stress in bone marrow-derived mesenchymal stem cells," Archives of Medical Research, vol. 44, no. 3, pp. 185-193, 2013.

[32] S. Y. Park, S. W. Lee, H. Y. Kim, W. S. Lee, K. W. Hong, and C. D. Kim, "HMGB1 induces angiogenesis in rheumatoid arthritis via HIF-1 $\alpha$ activation," European Journal of Immunology, vol. 45, no. 4, pp. 1216-1227, 2015. 

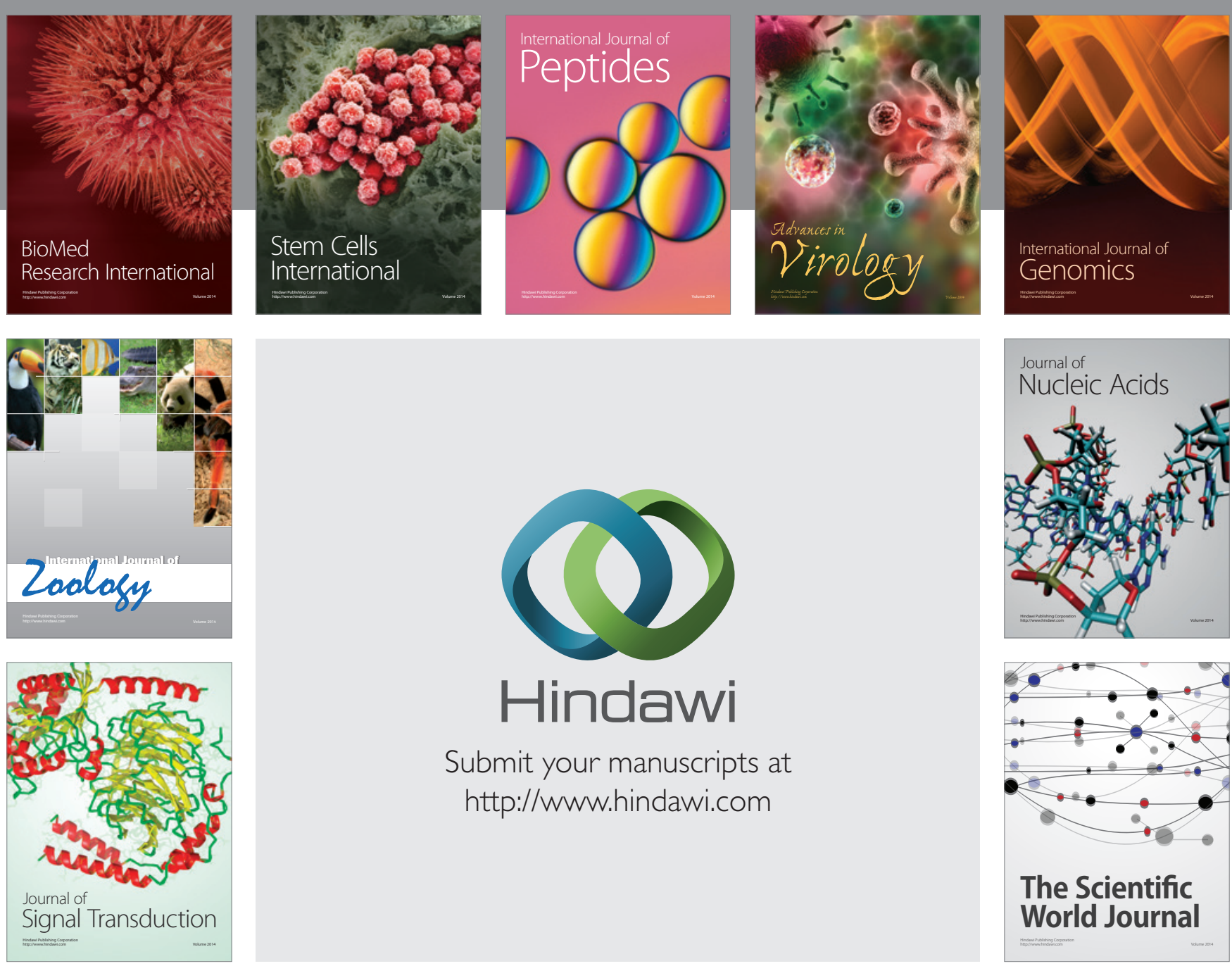

Submit your manuscripts at

http://www.hindawi.com
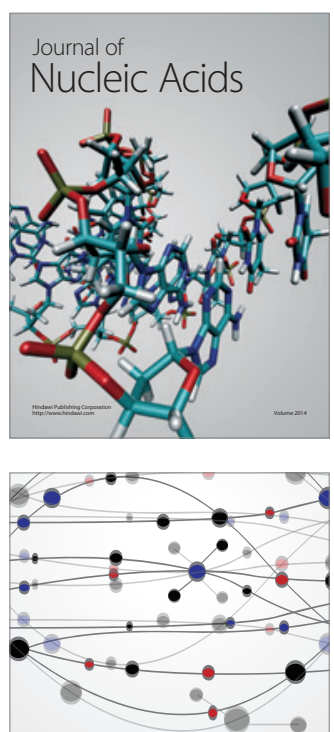

The Scientific World Journal
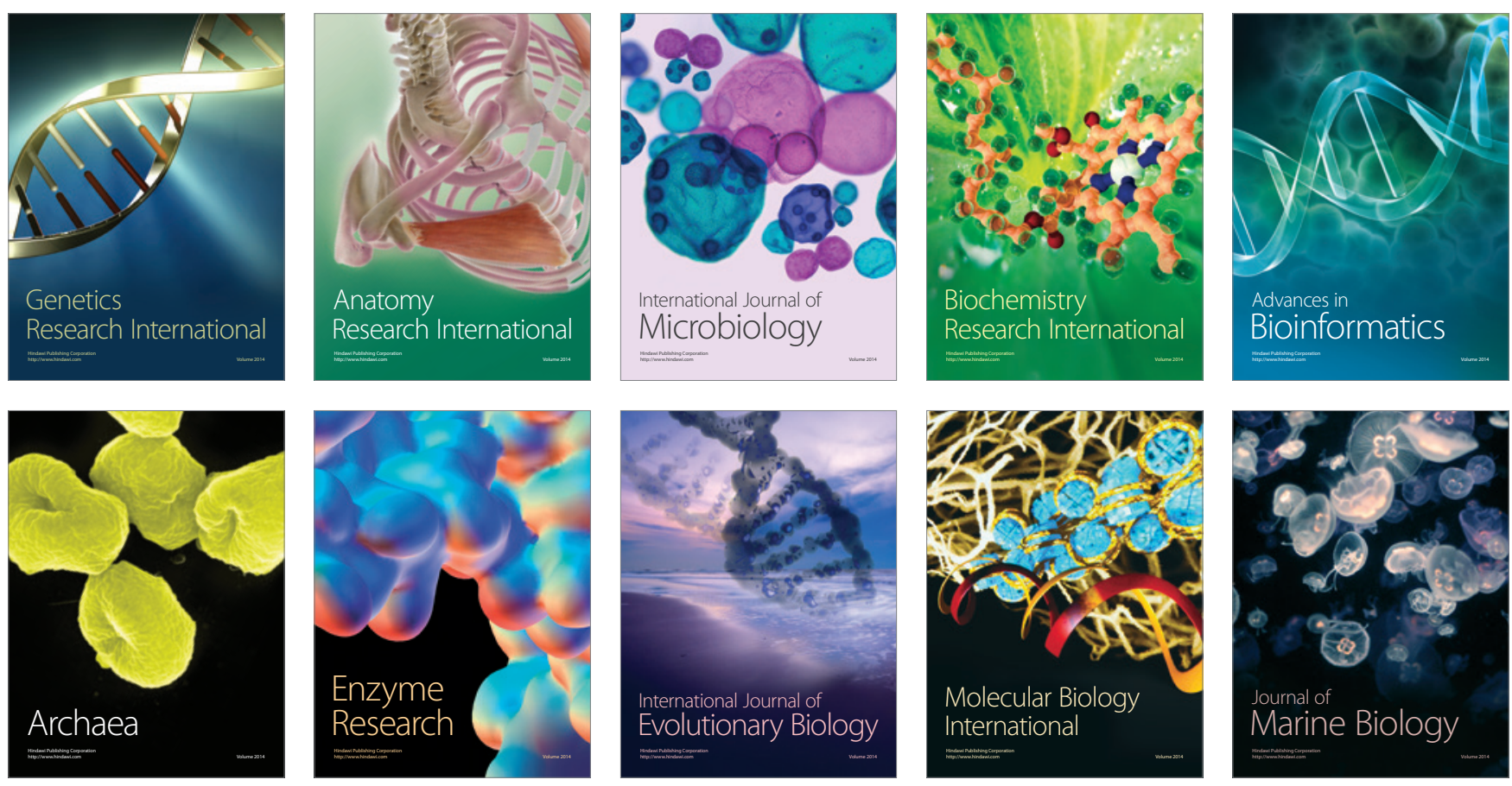